\title{
Réflexions sur la parole, le sacrifice et la mort dans quatre populations de l'Afrique de l'Ouest
}

Reflections on language, sacrifice and death among four populations of West Africa

\section{Germaine Dieterlen}

\section{OpenEdition}

\section{Journals}

Édition électronique

URL : http://journals.openedition.org/span/504

DOI : $10.4000 /$ span. 504

ISSN : 2268-1558

Éditeur

École pratique des hautes études. Sciences humaines

Édition imprimée

Date de publication : 30 mai 1981

Pagination : $61-70$

ISSN : 0294-7080

Référence électronique

Germaine Dieterlen, «Réflexions sur la parole, le sacrifice et la mort dans quatre populations de l'Afrique de l'Ouest », Systèmes de pensée en Afrique noire [En ligne], 5 | 1981, mis en ligne le 02 juillet 2013, consulté le 19 avril 2019. URL : http://journals.openedition.org/span/504 ; DOI : 10.4000/ span. 504

(c) École pratique des hautes études 
R E F L E X I O N S S U R L A P A R O L E ,

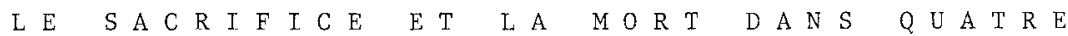
P O P U L A T I N S DE L'A F R I U E D E L'O E E T

par

Germaine Dieterlen

L'analyse de prières, d'invocations et de devises, recueillies avec leurs commentaires dans certaines populations d'Afrique de l'Ouest, nous a conduit à nous interroger sur les relations établies dans leur pensée entre la mort -- en particulier, la mort sacrificielle -- et la parole.

Comme nous $1^{\prime}$ avons dit dans une précédente étude sur les Dogon, pour ces derniers, tout sacrifice, quels qu'en soient le but et le mode opérationnel, réédite le sacrifice primordial du géniteur mythique de 1 'humanité, le Nommo (1). Comme dans son cas, tout sacrifice actuel implique un statut particulier des victimes. Il y a, en effet, une différence fondamentale entre le meurtre d'un animal, à la chasse par exemple, et la mise à mort sacrificielle. Le chasseur se protẽge au moyen d'amulettes et d'offrandes faites sur les autels de chasse des attaques de la force vitale (nyama), devenue vengeresse, des animaux occis. Le prêtre, en revanche, reste à l'abri des attaques dangereuses du nyama des victimes qu'il a fait sacrifier rituellement - immolations accompagnées de prières -- dans son sanctuaire. La mise à mort ne provoque pas de sentiment de culpabilité chez le sacrificateur, le sacrifiant ou les bẻnëficiaires du rite. Car tout sacrifice implique la prise en charge par la puissance invoquée, au moyen de paroles

(1) G. Dieterlen, Introduction à de nouvelles recherches sur le sacrifice chez les Dogon; Systèmes de pensée en Afrique noire, Cahier 2, Le sacrifice 1, 1976, p. 46. 
rituelles, des principes spirituels de la victime dont la vie se poursuivra en passant à un autre stade.

Le rôle du verbe est fondamental dans l'efficacité du rite sacrificiel : chaque opération est accompagnẻe d'une rêcitation, d'une prière, d'une invocation. Sans paroles le rituel n'a point d'effet; à la limite, c'est la parole qui compte. Dans une assemblée, on n'observe pas d'attitude particulière lorsque le sacrificateur procède aux libations ou à l'égorgement des victimes, il n'y a point de recueillement. En revanche, tout le monde se tait lorsque le doyen énonce -- et que le neveu utérin répète -- l'adresse, si courte soit-elle. Il se passe là quelque chose d'important dont tous les assistants ont pleinement conscience.

Sous des formes diffërentes, mais parallèlement, les mythologies des Bambara, des Malinké, des Dogon et đes Peul révèlent des associations particulières entre la parole et la mort, notamment la mort sacrificielle.

Parole et mort. Si l'on se réfère à la mythologie banbara, ce lien parâ̂t associé à une prise de conseience. Le mythe de la genèse relate que l'univers dans son ensemble fut rêalisé en plusieurs étapes par un Dieu, unique et créateur. Lors de la formation de la Terre, 1 'une de ses créatures, Pemba, descendit dans l'espace et l'occupa. Sous son règne, les hommes qui peuplèrent la planète "ignoraient le langage et le vêtement, vivaient nus et communiquaient entre eux par gestes et par grognements" (2). Ils étaient alors immortels, mais ils subissaient une autorité qui abusait de sa force. Pemba, qui avait pris la forme d'un arbre, le batanza, se nourrisait de leur sang et abusait sexuellement de leurs femmes; en contrepartie, les victimes étaient assurées d'un rajeunissement perpêtuel. Dieu dêpêcha alors sur la Terre une autre de ses premières créatures, Faro, mâttresse de l'eau, pour mettre un terme à cette exploitation. Il y eut entre eux colère et combat et Pemba, encore très puissant bien que vaincu,

(2) G. Dieterlen, Essai sur Za religion bambara, Paris, PUF, $1951, \mathrm{p} .17$. 
dëcida d'abandonner les hommes. Ceux-ci, dësespërés ne surent plus que faire, car ils pensaient que Pemba était leur géniteur et leur père. "Ils commencèrent à dépérir et leur patriarche se rendit auprès de 1'arbre pour implorer son pardon. "Il le pria vainement. Il se rendit alors près de 1 'eau et entendit la voix de Faro et à cause de la force de cette parole, il s'évanouit. Un peu d'eau le ranima, mais il fut effrayẻ par une ombre noire. Tendant les bras, il pria en disant : "L'arbre a mis l'obscurité entre toi et nous." Alors l'ombre devint blanche et la fraîcheur de Faro entra dans son corps. Il alla chercher les siens et Faro les instruisit, disant : "Je donnerai la pluie nécessaire aux cultures, les sources, les mares, les torrents, un grand fleuve, le Niger. Mais sachez que toute eau est sainte et doit être respectée. Je ne peux vous soustraire à la mort, mais j'y remédierai." Et il leur enseigna la parole et leur langue." Au premier d'entre eux, qui porta dès lors le nom de kumabana (litt. "il n'y a plus de paroles"), il dit : "Tu vas mourir". Ce dernier, sentant approcher sa fin, se rendit au bord de 1 'eau et dit à ceux qui 1 'entouraient : "Nous n'avions pas la parole; par la puissance de Faro, nous l'avons reçue. Voilà que maintenant il nous faut mourir. Je ne m'attendais pas à cette souffrance, à cette tristesse : tous vous connaîtrez la même." (3) A partir de ce moment, la mort apparut dans le genre humain, comme elle se produit encore aujourd'hui.

La mythologie malinkê insiste également sur la révélation de la parole aux premiers êtres humains (4). "Le troisième ancêtre, Simboumba Tagnagati, reçut de Faro trente premières paroles et huit graines de céréales dans la mare dans laquelle il pénétra après la chute de la première pluie sur 1a Terre. En sortant de l'eau, il dit : nko, "je parle". I1 construisit un sanctuaire dans lequel il mit les graines. A la porte de ce sanctuaire, Simboumba Tagnagati rểêla à ses frères

(3) G. Dieterlen, op. cit., pp. 24-25.

(4) G. Dieterlen, Mythe et organisation sociale au Soudan français, Journat de la Société des Africanistes, Tome XXV, Fasc. I et II, Paris, 1955, pp. 39-76.

Dans la mythologie malinké comme dans celle des Dogon, il s'agit de quatre frères qui descendirent avec leurs jumelles du ciel sur la Terre à bord d'une "arche" dirigée par leur géniteur mythique. Ils deviendront les ancêtres apicaux de 1 'humanité. 
les trente paroles de Faro; il parla toute la nuit et cessa de parler lorsqu'il vit se lever ensemble à l'horizon Sirius et le soleil". Après quoi la pluie tomba sur la terre, et les hommes délimitèrent le premier champ pour y semer les graines reçues. Plus tard, une autre série de paroles fut révêlée par Faro à Simboumba Tagnagati et fut également transmise aux hommes, après la victoire de Faro sur Pemba et Mousso Koroni, et l'extension de son pouvoir sur la terre. Cette transmission s'effectua au bord du Niger, I'ancêtre parlant sur une rive et le griot répétant sa parole en jouant du tambour sur l'autre. Simboumba Tagnagati prit alors le pouvoir "à cause de la parole". Le quatrième ancêtre rompit ensuite un interdit fondamental et mourut, ayant contrevenu aux instructions de Faro.

Le même schème apparaît dans les séquences de la mythologie dogon relatives à la parole. Le troisième ancêtre pénétra dans l'eau de la première mare où il reçut du Nommo, gêniteur mythique de 1 'humanitế (Faro chez les Malinké et les Bambara), la parole et l'enseignement. Il les révéla ensuite à ses frères; secondé par le griot. La récitation fut suivie de la consommation d'une bière faite du premier mil récolté, dans des conditions sur lesquelles nous reviendrons.

Cette séquencé a fait l'objet de représentations et d'aménagements dans une caverne de la région de Sanga. Une petite construction sise dans la caverne reprêsente le premier sanctuaire dans lequel 1 'ancêtre a parlè, tourné vers l'extêrieur. De l'autre côté d'un ruisseau temporaire qui coule en contrebas et représente symboliquement le Niger, des roches aménagées au long d'un escarpement évoquent le griot répétant les paroles, son tambour, les ancêtres qui l'écoutent et les astres qui se sont levés après cette révélation.

La même annêe, le quatrième ancêtre apprit un autre langage, celui qui avait été enseigné par le Nommo aux génies de brousse pour leur permettre de célêbrer les funérailles de l'un des leurs. Mais 1 'annêe suivante, 1 'ancêtre commit une très grave rupture d'interdit qui perturba l'ordre établi ; la terre devint sèche, la pluie cessa de tomber, il n'y eut plus de naissances parmi les hommes. Il fut alors sacrifié pour purifier la terre et mourut, une première fois. Le créateur, Amma, lui accorda cependant de ressusciter, comme c'êtait alors la règle, sous la forme d'un serpent. Mais le géniteur Nommo, qui 
siège dans les eaux terrestres, lui refusa l'une de ses âmes de sexe. Incomplet et stërile sous sa forme de serpent, il tenta de la lui demander, mais en vain ; il mourut cette fois-ci définitivement. Il fut alors éviré par ceux qui l'avaient sacrifié, pour remettre son sexe au géniteur et assurer ainsi une descendance à tous les hommes. La première version de cette séquence du mythe met l'accent sur la parole; elle doit retenir notre attention.

Les jeunes gens, qui n'avaient pas prévenu 1 'ancêtre-serpent, dansaient masqués .... "et descendaient vers le village. A cette vue, la colère du serpent déborda ; s'étant placé en travers du chemin que suivaient les masques, il leur fit en langue dogon les plus violents reproches, ce qui causa sa perte. Il avait en effet employé la langue des hommes, alors qu'il avait pris la forme d'un animal appartenant au monde des yebon. Devenu de ce fait impur pour eux, il ne pouvait plus vivre avec eux. Son nouvel état, d'autre part, l'empêchait de retourner chez les hommes. I1 mourut sur le champ" (5).

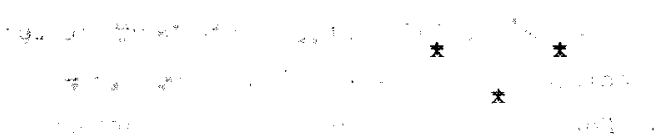

Sacrifice, mort et parole. Leur association se trouve exprimée dans un texte initiatique peul où est relaté l'enseignement donné au premier pasteur, Silé, par un personnage mythique, Koumen, gardien des troupeaux. L'instruction donnée à ce futur berger se poursuit pendant un voyage au cours duquel Silé s'arrête dans diverses "clairières" où son mâ̂tre lui parle. Dans la dernière clairière, Koumen énonce une longue incantation, adressée à Dialan, mâ̂tre des pâturages ; puis il dit à Silé : "Je vais te ramener à la lișière de mes domaines et $t$ 'y abandonner à tes propres forces ... tu vas avoir une lutte à mener contre un lion ... il porte entre les sourcils une touffe de poils ... tu le tueras ... pour cela, il te suffira de réciter l'incantation à Dialan et de frapper f'animal sur le nez ... il perdra connaissance et sera à ta merci ...tu l'égorgeras et tu le brûleras tout entier après avoir arraché la touffè de poils ...

(5) Cf. M. Griaule, Masques dogon, Paris, Institut d'Ethnologie, 1938, T. XXXIII, p. 62. Les yeban sont des génies qui occupent la brousse: les animaux sauvages sont leurs troupeaux. 
celle-ci sera cousue dans une bande de coton, de façon à confectionner un talisman ... ce dernier mis sous ta tête pendant son sommeil provoquera un rêve au cours duquel le vrai nom de la vache te sera révêlé par "l'esprit des eaux", pasteur des bovidés marins" (6).

Silé récite la litanie et c'est ella qui permet la mise à mort du lion en le mettant à sa merci. Il s'agit là clairement d'un sacrifice rituel ordonné pour parfaire une initiation, comportant une immolation associée à l'énoncé de paroles, rendue possible par un discours. I1 faut aussi souligner que l'enseignement initiatique se conclut et se résume à la fois par l'acquisition d'un nom -- celui du bovidé sacré -- "que le vieur berger souffle dans 1'oreille de Silé", nom que celui-ci soufflera à son tour dans l'oreille de son successeur quand le temps sera venu.

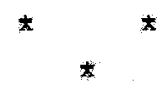

Il n'est pas question d'examiner ici toutes les valeurs et tous les sens que les diverses sociétés d'Afrique de 1 'Ouest réunissent sous le terme "parole" (7). Notre propos concerne les conceptions relatives à 1'oralité -- si fondamentale dans leurs traditions -- et, notamment, au discours contenant un enseignement susceptible d'agir dảns l'espace et le temps. Ce qui le distingue, bien entendu, de la conversation courante, du bavardage, du bruit et du cri, et même du rythme musical, encore que celui-ci soit considéré comme l'expression sonore d'un langage intellectuellement compris par les usagers.

Comme nous 1'avons relatê ci-dessus, de même que chez les Bambara et les Malinké, la mythologie dogon précise que la mort est apparue dans le monde humain après la révélation de la parole orale. Or, c'est aŷec sa "parole" que le Dieu créateur, Amma, a réalisé le monde en le dessinant d'abord au moyen de signes "abstraits", expressions premières et fondamentales de sa pensée créatrice. Ces signes, bummo, litt.

(6) Cf. A. Hampaté Ba et G. Dieterlen, Koumen, Texte initiatique des Pasteurs Peul, Cahiers de 1'Homme, Paris La Haye Mouton, 1961, pp. 81 à 91.

(7) Voir notamment G. Calame-Griaule, Ethnologie et Zangage : Za parole chez tes Dogon, Paris, Gallimard, 1965, $589 \mathrm{p}$. 
"traces", représentant cette parole, impliquent une manipulation des quatre éléments.

Le système graphique des Dogon présente une progression, en quatre étapes, de la forme du signe. La première est celle des signes abstraits dont nous venons de parler. La seconde comporte des signes faits de pointillês, dits yala, "marque" ou "image", qui évoquent le caractère de la chose représentée plutôt que sa forme. Les signes de la troisième étape, dits tônu. "schéma" ou "figure", sont faits de tracés non liés et représentent l'ébauche de l'objet. Enfin, la quatrième étape est celle des "dessins", liés et en couleur, qui se veulent réalistes et représentent l'objet ou l'être fini.

Lorsqu'on examine ces diverses figures dans une caverne ou sur la paroi d'un sanctuaire, on peut voir des exemples des trois dernières catégories. Les signes qui relèvent de la première catégorie sont tracés à 1'intérieur du sanctuaire, très rarement à 1'extérieur. La situation de ces diverses catégories de graphies et leur orientation sur les parois ont une valeur en soi : ce qui a été créé à l'intérieur du sein d'Amma est fait à l'intérieur, ce qui a été réalisé à l'extêrieur est reproduit à l'extérieur du sanctuaire et toujours orienté. Or qu'est-ce, pour les usagers, qu'un dessin réaliste? Il s'agit d'un être ou d'un objet achevé ; il est terminé, complet et "sorti", il va vers sa disparition dans le temps.

Il en est de mêtne de 1a parole. La parole orale est considêrée comme l'extériorisation de cette source de vie qui se trouvait aux origines dans le secret du sein d'Amma et qui, à cette étape, était silencieuse et créatrice. Lorsqu'elle est "sortie", complète et organisêe sous forme de discours, elle est allée vers une fin. Le mythe de la révélation du langage et de l'enseignement qu'il contient, implique, pour les hommes, la prise de conscience de leur situation dans l'espace et le temps et, partant, de la vie et de la mort. En contrepoint, soulignant cette représentation, le même mythe relate la déchéance du fauteur de désordre, Ogo. Transformé en renard, réduit au stade animal, il restera à jamais privé de la parole orale ; il vivra isolé, hors de la vie collective de ses "frères" mythiques et de leurs descendants.

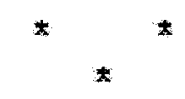


La séquence du mythe relative à la révélation de la parole met en évidence les liens établis entre parole, sacrifice et mort. Les cërémonies du Sigui, qui se déroulent sur le territoire dogon tous les soixante ans, commémorent cette révêlation, qui fut accompagnée de la consommation communielle de bière faite de mil rêcolté dans le premier champ collectif. Or, pour les Dogon, le traitement préalable auquel sont soumis les graines de mil, puis leur cuisson, connotent les étapes du sacrifice du Nommo. Nous rappelons ci-dessous ces relations symbaliques en: nous référant à une étude prêcédente (8).

-- "Faire tremper le grain" provoque la germination, soit la sortie du germe qui donnera naissance à une nouvelle plante. Cette opération technique est assimilée à celle qui fut réalisé avec le corps du Nommo dont le sexe fut d'abord tranchê en même temps que le cordon ombilical. Le sperme prélevé contenait I'eau de la pluie quí devait par la suite tomber régulièrement sur la terre, pour purifier le sol et permettre le développement de la vie animale et végétale ; il contenait aussi les germes des naissances futures dans le monde humain. -- "L"êtaler au soleil et le faire sêcher." Le grain, séparé de son germe, se dessèche et meurt, vidé de sa substance. C'est en plein soleil que le Nommo éviré fut ensuite égorgé et qu'il perdit à la fois son sang et ses symboles claviculaires.

-- "Ecraser le grain, le faire cuire pour préparer la bière", réédite le double travail d'Amra avec le corps de la victime; tout d'abord le découpage du corps en parcelles, pour la réorganisation de l'espace, parcelles dont les témoins sont les astres et les constellations; puis le regroupement des organes vitaux pour ensuite former un corps humain.

I1 faut ici evoquer, en les rêsumant, les représentations associées par les Dogon à la cuisson de la bière. Cette opération est assimilée à une manipulation des quatre éléments : 1'êlément terre est représenté par le contenant, une poterie ; 1 'eau par celle dans Iaquelle sont mises les graines concassées ; le feu par celui qui est entretenu sous le récipient ; l'air par celui qui s'échappe sous

(8) G. Dieterlen, Analyse d'une prière dogon, in L'autre et Z'ailleurs, (Homnage à Roger Bastide), Paris, Berger-Levrau1t, 1976, pp. 248 à 272 . 
forme de vapeur du liquide bouillant. Cette manipulation est symboliquement mise en parallèle avec celle qui fut inaugurêe par Amma, lequel brassa, mélangea les quatre éléments, pour ensuite former 1a graine primordiale, pó pizu, dans laquelle ils furent intégrés. Cuire le liquide réêdite 1 'une des étapes de la création du "germe" du monde. Le Créateur a manipulé les parties du corps du Nommo -- comme i1 1 'avait fait des quatre éléments -- pour réorganiser, c'est à dire remodeler 1'univers sur de nouvelles bases."

-- Mettre dans le liquide le support à ferment, lequel représente ici le "placenta d'Amma". Tressé en forme circulaire sur un moule, il supporte quatre pompons qui évoquent les quatre directions cardinales; assimilé à sa matrice, il représente la gestation du monde entier dans le sein d'Amma. Il est aussi le soleil issu de ce "placenta" initial auquel Amma a confié les travaux de fermentation. Le tremper dans la bière en prêparation qui vient de bouillir et dont les graines ont perdu toute vie, c'est remettre le soleil et ses rayons, ainsi que le monde orienté dans un milieu mort, mais prêt pour le renouveau et qu'il faut faire passer par les phases de la création. Le bouillonnement de la fermentation rappelie le moment où les êtres cherchaient à "sortir" de leur enveloppe.

De plus, le tressage de l'appareil rappelle celui du bonnet du chef religieux suprême des Dogon, réalisé lors de son intronisation (10). Il est fait des tiges de toutes les céréales cultivées et connote le moment où les hommes entrèrent en possession de ces graines. L'identitê porte-ferment - soleil - bonnet joue un rôle fondamental. C'est à cause d'elle que chaque semaine 1e Hogon d'Arou doit prêsenter son bonnet, dont le porte-ferment est le substitut, à l'est puis aux autres directions cardinales pour que 1 a bière fermente partout. Le Hogon "commande aux élêments de la bière, qui est affaire de chef et d'autorité, car la parole du chef qui l'a bue est celle du Nommo qui l'a révélée". Lors des cérémonies soixantenaires du Sigui, dont l'un des rites principaux est une consommation communielle de boisson fermentée, le Hogon d'Arou fait bouillir la première bière et en prélève

(9) G. Dieterlen, op. cit., p. 259.

(10) G. Dieterlen, Le titre d'honneur des Arou (sous presse). 
uné part qui est distribuée dans chaque famille pour être mélangée aux boissons particulières. Ainsi le chef marque-t-il sa préminence. Les participants au Sigui, après avoir entendu les "paroles" et reçu 1 'enseignement du Nommo, assis sur leurs crosses-sièges qui représentent son sexe fécond -- ils sont ses "enfants" -- boivent la bière quí, à elle seule, réédite son sacrifice et sa résurrection. En mệme temps, on commémore le décès de l'ancêtre en taillant une nouvelle image du serpent et en récitant son histoire dans la langue qu'il avait apprise, la "langue du Sigui". Ainsi sont intimement associêes, dans un rite magistral itinérant qui se déroule en pays dogon tous les soixante ans et pendant sept années consécutives, la révélation de la parole orale et l'apparition de la mort dans le monde humain. 